\title{
THE ASSOCIATION OF CELIAC DISEASE WITH OTHER AUTOIMMUNE DISEASES IN CHILDREN
}

\author{
Jelisaveta Maksimović ${ }^{1}$, Zlatko Djurić ${ }^{1,2}$ \\ ${ }^{1}$ Faculty of Medicine, University of Niš, Niš, Serbia \\ ${ }^{2}$ Children's Hospital, Department of Gastroenterology, Niš, Serbia
}

\begin{abstract}
Celiac disease (CD) is an autoimmune disease with estimated prevalence of $1 \%$ in European and North American population. All four components of CD autoimmunity are well known, such as: genetics, autoantigen, autoantibodies and the environmental triggers. Besides gastrointestinal manifestations, celiac disease has a wide array of extraintestinal symptoms and clinical signs. This article briefly summarizes celiac disease pathophysiology and association of celiac disease with other autoimmune diseases in children. It puts emphasis on possible protective role of gluten-free diet for the development of other autoimmune diseases in patients with celiac disease.
\end{abstract}

Key words: celiac disease, autoimmune diseases, diabetes mellitus.

\section{Introduction}

Over the last few decades increased prevalence of many autoimmune diseases in the human population has been documented. Celiac disease (CD) is one of the most common autoimmune diseases in human population and the only disease with well-known environmental triggers of autoimmunity. Patients with one autoimmune disease are more susceptible to other autoimmune diseases. Coexistence of two or more autoimmune diseases in the same individual (autoimmune polyendocrine syndrome) has been published in literature [1].

At the beginning of the twentieth century, with the advent of the highly sensitive and highly specific serological screening tests for $\mathrm{CD}$, large screening studies have been put into practice with the aim of identifying CD prevalence in general population and patients suffering from other autoimmune diseases.

\section{Celiac Disease}

$\mathrm{CD}$ is common, lifelong disease characterized by autoimmune damage of the small bowel mucosa. Recent epidemiological studies indicate that $\mathrm{CD}$ affects about $1 \%$ of European and North American population [2,3]. Nowadays, all 4 components of CD autoimmunity are well known, such as: genetics, autoantigen, autoantibodies and the environmental triggers. Genetic predisposition is needed for CD occurrence. More than $95 \%$ of patients

* Correspondence to: Jelisaveta Maksimović, M.D.

Faculty of Medicine, University of Niš, Bulevar dr Zorana Đindjića 81, 18000 Niš, Serbia

Phone: +3810603010190

E-mail: saveta17@gmail.com

Received July $7^{\text {th }}, 2017$, accepted for publication September $5^{\text {th }}, 2017$ are carriers of HLA-DQ2 haplotype (encoded by alleles DQA1 * 05 and DQB1 * 02). The rest of the patients are carriers of HLA-DQ8 (encoded by alleles DQA1 * 03 and $*$ DQB1- 0302). The role of the genes out of the HLA system was examined by GWARS (genome-wide association studies) [4,5]. More than 115 of these genes have been involved in the regulation of the innate and adaptive immune responses. However, the importance of each individual gene outside the HLA system for the development of CD is very small.

The enzyme tissue transglutaminase (TG2) is located in lamina propria of small bowel mucosa. TG2 leads to deamination of glutamine residue of gliadin [6]. This reaction provides glutamine residue a negative charge and ability to bind to HLA DQ2 / DQ8 molecule on the antigen-presenting cells. TG2 participates in many physiological processes, such as extracellular matrix building, tissue reparation, signal mechanisms of receptors, cell proliferation, cell motility and endocytosis [7]. In addition to the small intestine, TG2 can also be found in the liver, muscles, and lymph nodes.

Dietrich et al. (1997) were the first who discovered IgA antibodies against TG2 (Anti-TG2) in the sera of CD patients [8]. Deployment of tests based on AntiTG2 detection substantially improved the diagnostic accuracy of CD serologic testing.

Development of $\mathrm{CD}$ requires food intake that contains gliadin fraction of gluten from wheat or similar prolamins from rye (secalins) and barley (hordeins). There are gliadin peptides which demonstrate two different effects on small bowel mucosa: toxic and immunostimulatory [9]. Alpha-gliadin peptides (31-43, 44-55, 56-75) with toxic activity are responsible for the damage of the small bowel mucosa and initiation of the pathophysiological processes in CD. Peptides with 
immunostimulatory effects initiate $\mathrm{T}$ helper 1 (Th1) response and secretion of interferon- $\gamma$. It is assumed that there are more than 50 gliadin peptides with this kind of effect.

The pathophysiology of autoimmune damage of small bowel mucosa in CD is not completely understood. It encompasses complex interactions between the innate and adaptive immune response, triggered by ingestion of gluten. At the beginning of this process paracellular permeability of small bowel mucosa is increased for gluten peptides due to peptide induced CXCR3 activated upregulation of zonulin, an intestinal peptide involved in tight junctions control [10].

Toxic gliadin peptides activate the innate immune system and increase production of interleukin 15 (IL-15) by epithelial and dendritic cells. IL-15 stimulates cytotoxic activity of intraepithelial lymphocytes through production of interferon $\gamma$ (IFN $\gamma$ ) and stimulation of NKG2D cells. Activation of the adaptive immune response occurs after deamination of glutamine residues with TG2, their binding to dendritic cells and presentation to CD4 T lymphocytes followed by activation of the cellular (Th1) and humoral (Th2) immune response. Th1 response leads to the IFN $\gamma$ induced cell death of enterocytes. Th2 response causes differentiation of $\mathrm{B}$ lymphocytes into plasma cells capable of producing antiTG2 and antigliadin antibodies (AGA). It is not known whether anti-TG2 in a certain way influences the activity of the TG2. In addition to Anti-TG2 other types of autoantibodies, such as antibodies against actin and reticulin were detected in CD [11].

CD may be manifested by typical (chronic diarrhea, loss of appetite, distended abdomen, malnutrition) and non-typical symptoms (stunted growth, refractory anemia, osteoporosis, unexplained hypertransaminasemia). The CD diagnosis is established on the basis of positive serology (anti-TG2 or anti-endomysial antibodies) and small bowel mucosa pathohistology.

\section{Celiac Disease and Other Autoimmune Diseases}

A group of autoimmune diseases is consisted of about 80 different diseases affecting $5-8 \%$ of the human population [12]. Patients with a single autoimmune disease are at $25 \%$ risk of development of other autoimmune diseases [13]. Many studies in children have reported an association between $\mathrm{CD}$ and various autoimmune diseases. Common genetic background is one of the predisposing factors for this association $[14,15]$. There is a possibility that persistent activation by proinflammatory cytokines leads to the unmasking of other autoantigens (besides TG2) and the initiation of a new autoimmune attack. Recent studies demonstrated that gut microflora plays an important role, not only in shaping the immune responses, but also in the development of autoimmunity [16].

Italian authors have found an increased frequency of organ-specific antibodies in CD patients [17]. Titer of antibodies significantly decreased following the commencement of the gluten-free diet (GFD). On the basis of this, they hypothesized that GFD may prevent the occurrence of other autoimmune diseases. However, subsequent studies have not confirmed the protective role of GFD $[18,19]$. Development of other autoimmune disease is more likely if the $\mathrm{CD}$ is diagnosed in young individuals with positive family history of CD [20].

\section{Celiac Disease and Diabetes Mellitus Type 1}

Diabetes mellitus type 1 (DMT1) is one of the most common chronic diseases in children with increasing annual prevalence from $3 \%$ to $4 \%$ [21]. Numerous screening studies conducted around the world showed the increased prevalence of $\mathrm{CD}(2.4 \%-16.4 \%)$ in patients with DMT1 [22]. The only screening study of this type in Serbia revealed the CD prevalence of $5.79 \%$ in children and adolescents with DMT1 [23].

DMT1 patients with CD can be asymptomatic (60\%$70 \%$ of patients) or have classical CD symptoms. It should be kept in mind that positive titer of anti-TG2 in DMT1 patients does not always mean that they have CD. In a number of serologically positive DMT1 patients who do not have symptoms of the $\mathrm{CD}$, antiTG2 disappeared spontaneously, even though they continued to use gluten containing food. In these children anti-TG2 titer levels are usually slightly lower than in asymptomatic CD patients.

Some studies suggest that patients with newly diagnosed DMT1 and CD have frequent hypoglycemic episodes, higher average value of glycosylated hemoglobin, and reduced bone mineral density [24-26]. Conflicting evidence exist as to whether a GFD significantly improves glycemic control in DMT1 patients $[24,27,28]$.

Children and adolescents with DMT1 and CD are prone to vascular complications later in life. $\mathrm{CD}$ is regarded as an independent risk factor for retinopathy and nephropathy in patients with DMT1 [29].

The International Society for Pediatric and Adolescent Diabetes (ISPAD) recommended serological screening for CD in all children with DMT1 at the time of DMT1 diagnosis and every 1-2 years thereafter [30] because positive titer of Anti-TG2 can be found many years after the beginning of DMT1.

\section{Celiac Disease and Autoimmune Thyroid Disease}

Many screening studies in children with autoimmune thyroid disease (Hashimoto's thyroiditis, Graves' disease) have found an increased prevalence of CD (2.3-7.9\%) [31]. CD has been more commonly found in autoimmune thyroiditis than in Graves' disease. Serologic CD screening is recommended in children with autoimmune thyroid disease by the European Society for Pediatric Gastroenterology, Hepatology and Nutrition (ESPGHAN) [32]. 
Investigations were carried out in the opposite direction as well, with the aim of determining the prevalence of autoimmune thyroid disease in children with $\mathrm{CD}$. In one Italian study autoimmune thyroid disease was found in $26.2 \%$ of pediatric patients with CD [33]. Thyroid dysfunction in $C D$ has a negative impact not only on growth, metabolism of proteins and cholesterol, but also on cognitive function and fertility.

\section{Celiac Disease and Autoimmune Hepatitis}

In case of elevated transaminases in children with $\mathrm{CD}$, autoimmune hepatitis and non-specific reactive hepatitis (celiac hepatitis) should be considered. Autoimmune hepatitis (AIH) is a chronic disease in genetically predisposed individuals in whom the autoimmunity is directed toward liver antigens. Pediatric surveys have reported a wide prevalence of $\mathrm{CD}$ in $\mathrm{AIH}$ in a range from $3.6 \%$ to $12 \%$ [34]. Although there are still no recommendations for routine serological $\mathrm{CD}$ screening in patients with $\mathrm{AIH}$, the association between these two diseases should be considered if one of them is diagnosed. Rare cases of $\mathrm{CD}$ associated with other autoimmune liver diseases such as primary sclerosing cholangitis (PSC) and "overlap" syndrome (AIH / PSC) were described in children $[35,36]$.

Celiac hepatitis is found in $26-57 \%$ of children with CD [37,38]. It is manifested by the elevation of transaminases without any symptoms of liver disease. The increased intestinal permeability in CD may ease the entry of toxins, antigens, antibodies and cytokines to the portal system leading to hepatocytes function impairment [34]. In most cases (70-100\%) transaminase levels are normalized within a year on GFD. All patients with unexplained hypertransaminasemia should be serologically tested for CD.

\section{Celiac Disease and Dermatological Diseases}

CD may be associated with various skin manifestations, such as dermatitis herpetiformis (DH), psoriasis, alopecia

\section{References}

1. Kakleas K, Soldatou A, Karachaliou F, Karavanaki K. Associated autoimmune diseases in children and adolescents with type 1 diabetes mellitus (T1DM). Autoimmun Rev 2015; 14:781-797.

2. Mustahalti K, Catassi C, Reunanen A, et al. The prevalence of celiac disease in Europe: results of centralized, international mass screening project. Ann Med 2010; 42:587-595.

3. Fasano A, Berti I, Geralduzzi T, Not T, Colletti RB, et al. Prevalence of celiac disease in at-risk and not-at risk groups in the United States: a large multicenter study. Arch Intern Med 2003; 163:286-292.

4. Garner CP, Murray JA, Ding YC, et al. Replication of celiac disease UK genome-wide association study results in a US population. Hum Mol Genet 2009; 18:4219-4225.

5. Trynka G, Zhernakova A, Romanos J, et al. Coeliac disease associated risk variants in TNFAIP3 and REL implicate altered NF-kappaB signaling. Gut 2009; 58:1078-1083. areata and vitiligo [39-41]. DH is most frequently found in adolescents, young and middle-aged adults. All patients with $\mathrm{DH}$ have some forms of enteropathy that can be found in CD. In $10-20 \%$ of $\mathrm{DH}$ patients, in addition to rash, there is a typical clinical picture of a CD while in the rest of $80-90 \%$ atypical or "silent" CD clinical forms are seen. GFD is standard treatment for DH with excellent clinical response. Since the reintroduction of gluten in a diet after clinical recovery on GFD triggers relapses of cutaneous and intestinal lesions, $\mathrm{DH}$ is regarded as skin manifestation of CD [39].

\section{Celiac disease and rheumatological diseases}

$\mathrm{CD}$ is found in in $1.5 \%-2.8 \%$ of children with juvenile rheumatoid arthritis $[42,43]$. The prevalence of CD is less studied in other rheumatological diseases in pediatric population. Studies examining positive effect of GFD on arthritis were not conducted in children, while in adults they are very rare. Sjogren's syndrome is considered the most common rheumatological disease associated with CD (in $12 \%-14.4 \%$ cases) in adults $[44,45]$.

\section{Conclusion}

CD may be associated with many diseases of autoimmune origin. Currently, there are recommendations of international societies such as ISPAD and ESPGHAN for CD serological screening only in children with T1DM and autoimmune thyroid disease. Future studies with the involvement of large number of children, including a cost-benefit analysis, are going to show whether CD screening for children with other autoimmune diseases will be justified. Despite a very promising hypothesis, there is still no scientifically based evidence that GFD has prophylactic effect on the development of other autoimmune diseases in patients with CD.

6. Solid LM. Coeliac disease: dissecting a complex inflammatory disorder. Nature 2002; 2:647-655.

7. Troncone R, Discepolo V. Celiac disease and autoimmunity. J Pediatr Gastroenterol Nutr 2014; 59 Suppl 11:S9-S11.

8. Dietrich W, Ehnis T, Bauer M, et al. Identification of tissue transglutaminase as the autoantigen of celiac disesase. Nat Med 1997; 3:797-801.

9. Hekkens WTJM, Haex AJC, Willighagen RGJ. Some aspects of gliadin fractionation and testing by histochemical method. In: Booth CC, Dowling RH. Celiac disease. Edinburgh: Churcil Livingstone 1970:11-19.

10. Lammers $\mathrm{KM}, \mathrm{Lu} \mathrm{R}$, Brownley $\mathrm{J}$, et al. Gliadin induces an increase in intestinal permeability and zonulin release by binding to the chemokine receptor CXCR3. Gastroenterology 2008; 135:194-204.

11. Seah PP, Fry L, Rossiter MA, Hoffbrand AV, Holborow EJ. Anti-reticulin antibodies in childhood coeliac disease. Lancet $1971 ; 2: 681-682$. 
12. Dragin N, Le Panse R, Berrih-Aknin S. Autoimmune disease predisposition: Aire «protects» men. Med Sci (Paris) 2017; 33 : 169-175.

13. Harpereet S, Deepak J, Kiran B, Multiple autoimmune syndrome with celiac disease. Reumatologia 2016; 54:326-329.

14. Bardella MT, Elli L, De Matteis S, Floriani I, Torri V, Piodi L. Autoimmune disorders in patients affected by celiac sprue and inflammatory bowel disease. Ann Med 2009; 41:139-143.

15. Elli L, Bonura A, Garavaglia D, Rulli E, Floriani I, Tagliabue G, Contiero P, Bardella MT. Immunological comorbity in coeliac disease: associations, risk factors and clinical implications. J Clin Immunol 2012; 32:984-990.

16. Cenit MC, Olivares M, Codoner-Franch P, Sanz Y. Intestinal microbiota and celiac disease: cause, consequence and coevolution? Nutritiens 2015; 7:6900-6923.

17. Ventura A, Magazzu G, Greco L. Duration of exposure to gliadin and risk for autoimmune disorders in patients with celiac disease, Study group for autoimmune disorders in celiac disease. Gastroenterology 1999; 117:297-303.

18. Hummel S, Pfluger M, Hummel M, Bonifacio E, Ziegler AG. Primary dietary intervention study to reduce the risk of islet autoimmunity in children at increased risk for type 1 diabetes: the BABYDIET study. Diabetes Care 2012; 47:43-48.

19. Metso S. Gluten free diet and autoimmune thyroiditis in patients with celiac disease. A prospective controlled study. Scand J Gastroenterol 2012; 47:43-48.

20. Cosnes J, Celler C, Viola S, et al. Incidence of autoimmune diseases in celiac disease: protective effect of gluten- free diet. Clin Gastroenterol Hepatol 2008; 6:753-758.

21. Patterson CC, Gyurus E, Rosenbauer $J$, et al. Trends in childhood type 1 diabetes incidence in Europe during 19892008: evidence of non-uniformity over time in rates of increase. Diabetologia 2012; 55:2142-2147.

22. Holmes GKT. Screeinig for coeliac disease in type 1 diabetes. Arch Dis Child 2002; 87:495-499.

23. Djuric Z, Stamenkovic H, Stankovic T, Milicevic R, Brankovic L, Ciric V, Katic V. Celiac disease prevalence in children and adolescents with type 1 diabetes form Serbia. Pediatr Int 2010; 52:579-583

24. Goh C, Banerjee K. Prevalence of coeliac disease in children and adolescents with type 1 diabetes mellitus in clinic based population. Postgrad Med J 2007; 83:132-136

25. Iafusco D, Rea F, Prisco F. Hypoglycemia and reduction of insulin requirement as a sign of celiac disease in children with IDDM. Diabetes Care 1998; 21:1379-1381.

26. Simmons KM, McFann K, Taki I, Liu E, Klingensmith GJ, Rewers MJ, Frohnert BI. Reduced bone mineral density is associated with celiac disease autoimmunity in children with type 1 diabetes. J Pediatr 2016; 169:44-48

27. Kaukinen K, Salmi J, Lahtela J, Siljamäki-Ojansuu U, Koivisto AM, Oksa H, Collin P No effect of gluten free diet on the metabolic control of type 1 diabetes in patients with diabetes and celiac disease, Retrospective and controlled prospective survey. Diabetes Care 1999; 22:1747-1748.

28. Kaspers S, Kordonouri O, Schober E, Grabert M, Hauffa BP, Holl RW; German Working Group for Pediatric Diabetology. Anthropometry, metabolic control and thyroid autoimmunity in type 1 diabetes with celiac disease: a multicenter survey. J Pediatr 2004; 14:790-795.
29. Rohrer TR, Wolf J, Liptay S, Zimmer KP, et al. DPV Initiative and the German BMBF Competence Network Diabetes Mellitus. Microvascular complications in childhood-onset type 1 diabetes and celiac disease: A multicenter longitudinal analysis of 56,514 patients from the German-Austrian DPV database. Diabetes Care 2015; 38:801-807.

30. Kordonouri O, Klingensmith G, Knip M, Holl RW, Menon PSN, Aanstoot HJ, Craig ME; International Society for Pediatric and Adolescent Diabetes. ISPAD clinical practice consensus guidelines 2014. Other complications and diabetes associated conditions in children and adolescents. Pediatr Diabetes 2014; 15 Suppl 20: 270-278.

31. Sattar N, Lazare F, Kacer M, et al. Celiac disease in children, adolescents, and young adults with autoimmune thyroid disease. $\mathrm{J}$ Pediatr 2011; 158:272-273.

32. Husby S, Koletzko, S, Karponay-Szabo IR, et al. European society for pediatric gastroenterology, hepatology and nutrition guidelines for the diagnosis of celiac disease. J Pediatr Gastroenterol Nutr 2012; 54:136-160.

33. Ansaldi N, Palmas T, Corrias A, Barbato M, et al. Autoimmune thyroid disease in children. J Pediatr Gastroenterol Nutr 2003 37:63-66.

34. Anania C, De Lucia E, De Castro G, Chiesa C, Pacifico L. Liver involment in pediatric celiac disease. World J Gastroenterol 2015; 21:5813-5822.

35. Deneau M, Jensen MK, Holmen J, Williams MS, Book LS, Guthery SL. Primary sclerosing cholangitis, autoimmune hepatitis, and overlap in Utah children: epidemiology and natural history. Hepatology 2013; 58:1392-1400.

36. Gregorio GV, Portman B, Karani J, Harrison P, Donaldson PT, Vergani D, Mieli-Vergani G. Autoimmune hepatitis/sclerosing cholangitis overlap syndrome in childhood: a 16-year prospective study. Hepatology 2001; 33:544-553.

37. Arslan N, Bayukgebiz B, Ozturk Y, Ozer E. The prevalence of liver function abnormalities in pediatric celiac disease patients and its relation with intestinal biopsy findings. Acta Gastroenterol Belg 2005; 68:424-427.

38. Bonamico M, Pitzalis G, Culasso F, Vania A, Monti S, Benedetti C, Mariani P, SignorettiA. [Hepatic damage in celiac disease in children]. Minerva Pediatr 1986; 38:959-962.

39. Pinto-Sanchez MI, Berick P, Verdu EF, Bai JC. Extraintestinal manifestations of celiac disease. Dig Dis 2015; 33:147-154.

40. Ludvigsson JF, Lindelof B, Zingone F, Ciacci C. Psoriasis in a nationwide cohort study of patients with celiac disease. J Invest Dermatol 2011; 131:2010-2016.

41. Fessatou S, Kostaki M, Karpathios T. Coeliac disease and alopecia areata in childhood. J Pediatr Child Health 2003; 39:152-154.

42. George EK, Mearin ML, Bourquet J, et al. Screeninig for coeliac disease in Duch children with associated diseases. Acta Paediatr Suppl 1996; 412:52-53.

43. Lepore L, Martelossi S, Pennesi M, Falcini F, Ermini ML, et al. Prevalence of celiac disease in patients with juvenile chronic arthritis. J Pediatr 1996; 129:311-313.

44. Iltanen S, Collin P, Korpela M, Holm K, Partanen J, Polvi A, Maki M. Celiac disease and markers of celiac disease latency in patients with primary Sjogren's syndrome. Am J Gastroenterol 1999; 94:1042-1046.

45. Luft LM, Barr SG, Martin LO, Chan EK, Fritzler MJ. Antibodies to tissue transglutaminase in Sjogren's syndrome and related rheumatic diseases. J Rheumatol 2003; 30:2613-2619. 